\title{
How nuclear data collected for medical radionuclides production could constrain nuclear codes
}

\author{
Arnaud Guertin ${ }^{1, \text { a }}$, Charlotte Duchemin ${ }^{1}$, Arnaud Fardin ${ }^{1}$, Corentin Guigot ${ }^{1}$, Etienne Nigron ${ }^{1}$, Charlotte Remy ${ }^{1}$, \\ Férid Haddad $^{1,2}$, Nathalie Michel ${ }^{1,2}$, and Vincent Métivier ${ }^{1}$ \\ 1 Laboratoire SUBATECH, CNRS/IN2P3, Ecole des Mines de Nantes, Université de Nantes, 4, rue Alfred Kastler, \\ 44307 Nantes, France \\ 2 GIP ARRONAX, 1 rue Aronnax, 44817 Saint Herblain, France
}

\begin{abstract}
The aim of this article is to give the status of nuclear data collected in the framework of the production of innovative radionuclides for therapy and diagnosis applications in collaboration with the GIP ARRONAX, which possesses a multi-particle high energy cyclotron, and to show constrains that can be put on nuclear codes. In order to assess the cross section of a given production route, experiments have been carried out using the stacked-foil technique and gamma-spectroscopy for a set of radionuclides of medical interest: photon (Tc-99m) and positon (Sc-44g) emitters for diagnosis, electron (Re-186g, Tb-155, Sn-117m) and alpha particle (Th-226, Ra-233, Bi-213) emitters for therapeutic applications. A systematic comparison has been performed between the results from the TALYS code (version 1.6) and the large set of data collected using different projectiles (proton, deuteron and alpha particle) from few $\mathrm{MeV}$ up to $70 \mathrm{MeV}$ and covering a wide range of target masses. A better overall agreement with our experimental data has been obtained with a combination of models already included in the TALYS code differing from the default one.
\end{abstract}

\section{Introduction}

Our research activities are focused on radionuclide production mainly for medical applications, either for therapy or diagnosis. This work is carried out in close collaboration with the GIP ARRONAX that possesses a high energy and high intensity multi-particle cyclotron [1].

In this frame, production cross sections and thick target yields were measured for alpha emitters, such as the U-230/Th-226, Th-227/Ra-223 and Ac-225/ Bi-213 generators [2]; for photon, Tc-99m [2], and positon, Sc-44g [3], emitters for diagnosis; for electron emitters Re-186g [4], Tb-155 [5] and Sn-117m [6] for therapeutic applications. From the irradiated materials, new experimental production cross section data of interest for medical applications and monitor reactions have been extracted which allow to expand our knowledge on these excitation functions, to confirm the existing trends and to give additional values on a wider energy range. These experiments were conducted at the ARRONAX facility using the stacked-foil technique over the four last years.

This data set only represents a small part of the data needed in the field studying the production of innovative radionuclides for medical applications. In order to get answers quickly without the need of new experiments, it is interesting to use theoretical models. The TALYS code gather together several of such models for each step of a nuclear interaction.

A systematic comparison of our results with the output of the TENDL-2015 nuclear data library and TALYS code has been done. In this latter case, several combinations of

a e-mail: Arnaud.Guertin@subatech.in2p3.fr models have been tested in order to better reproduce the available data.

\section{Material and methods}

\subsection{Experimental set-up}

The production cross section data are obtained using the stacked-foil method [2,7], which consists of the irradiation of a set of thin foils, grouped as patterns. Each pattern contains a target to produce the isotopes of interest. Each target is followed by a monitor foil to have information on the beam intensity thanks to the use of a reference reaction recommended by the International Atomic Energy Agency [8]. A degrader foil is placed after each monitor foil to change the incident beam energy from one target foil to the next one. Each foil in the stack is weighed before irradiation using an accurate scale $\left( \pm 10^{-5} \mathrm{~g}\right)$ and scanned to precisely determine its area. The thickness is deduced from these values, assuming that it is homogeneous over the whole surface. In this work, we used thin target and monitor foils of a few tens of micrometres thick and degrader foils of few hundreds of micrometres thick. All foils were purchased from the Good Fellow(c)company.

These foils were irradiated by proton (up to $70 \mathrm{MeV}$ ), deuteron (up to $34 \mathrm{MeV}$ ) or alpha particle $(68 \mathrm{MeV})$ beams provided by the ARRONAX cyclotron. It delivers these beams with an energy uncertainty of $\pm 0.50 \mathrm{MeV}$, $\pm 0.25 \mathrm{MeV}$ and $\pm 0.61 \mathrm{MeV}$, respectively for $\mathrm{p}$, $\mathrm{d}$ or alpha particle, as specified by the cyclotron provider using simulations. The beam line is under vacuum and closed using a $75 \mu \mathrm{m}$ thick kapton foil. The stacks were located about $6.8 \mathrm{~cm}$ downstream in air. 
The energy through each foil has been determined in the middle of the thickness of the foil using the SRIM software [9]. Energy losses in the kapton foil and air have been taken into account in our analysis. All along the stack, depending on the number of foils, the energy uncertainty increases up to $\pm 2.0 \mathrm{MeV}$ due to the energy straggling. Irradiations were usually carried out for half an hour, with beams of mean intensity between 100 and $150 \mathrm{nA}$ for proton, between 50 and $140 \mathrm{nA}$ for deuteron and between 140 and $200 \mathrm{nA}$ particles for alpha beam.

The recommended production cross section values [7] of the Ti-nat(d, x)V-48 (all energies), Cu-nat(p,x) Co-56, Zn-62 (> 50 MeV), Ti-nat(p,x)V-48 (<20 MeV), Ni-nat(p,x)Ni-57 (20-50 MeV), Cu-nat $(\alpha, x)$ Ga-67 (up to $50 \mathrm{MeV}$ ) and $\mathrm{Al}-27(\alpha, \mathrm{x}) \mathrm{Na}-22$ (from $50 \mathrm{MeV}$ to $70 \mathrm{MeV}$ ) reactions were used to get information on the beam intensity, depending on the investigated energy range.

The activity measurements in each foil were performed using a high purity germanium detector from Canberra (France) with low-background lead and copper shielding. The first measurements started the day after the irradiation (after a minimum of 15 hours cooling time) during one hour, for all target and monitor foils. Our data are then limited to $\gamma$ emitter radionuclides with a half-life higher than few hours. A second series of measurements was performed one week after End Of Beam, during a minimum of 24 hours (one day) and up to 60 hours. Third measurements were devoted to long half-life radionuclides and also waiting for the decay of some radionuclides.

Gamma spectra were recorded in a suitable geometry calibrated in energy and efficiency with standard Co-57, Co-60 and Eu-152 gamma sources from LEA-CERCA (France). The full widths at half maxima were $1.04 \mathrm{keV}$ at $122 \mathrm{keV}(\mathrm{Co}-57 \gamma$ ray) and $1.97 \mathrm{keV}$ at $1332 \mathrm{keV}(\mathrm{Co}-60 \gamma$ ray). The samples were placed at a distance of $19 \mathrm{~cm}$ from the detector, which is suited to reduce the dead time and the effect of sum peaks. The dead time during the counting was always kept below $10 \%$.

\subsection{Production cross section calculation}

The production cross section values are calculated using the well-known activation formula, defined as a relative equation in which the knowledge of the beam current is no longer necessary thanks to the recommended reactions. The uncertainty is expressed as a propagation error calculation (see [6] for more details).

\subsection{The TALYS code and TENDL data library}

In this work, all the experimental production cross section values are compared with the version 1.6 of the TALYS code released in December, 2013 [10]. TALYS is a nuclear reaction program which simulates reactions induced by light particles on nuclei ranging from $\mathrm{Li}$ to $\mathrm{Ds}$. It incorporates theoretical models to predict observables including production cross section values as a function of the incident particle energy (up to $1 \mathrm{GeV}$ ). A combination of models that best describes the whole set of available data for all projectiles, targets and incident energies have been defined by the authors and put as default in the code. In this way, a calculation can be performed with minimum information in the input file: the type of projectile and its incident energy, the target type and its mass. The results of this combination of models are referenced in Figs. 1 to 4 as TALYS 1.6 Default.

TENDL is a TALYS-based Evaluated Nuclear Data Library using both default and adjusted TALYS calculations and data from other sources [10,11]. Our experimental production cross section values have been compared with the $8^{\text {th }}$ TENDL release: TENDL-2015. It provides evaluated data for seven types of incident particles (n, p, d, t, He-3, alpha-particle, gamma) and for all the isotopes living more than 1 second ( 2800 isotopes), up to $200 \mathrm{MeV}$.

Since there are some differences between experimental data and the results of the TALYS code using default models and the TENDL-2015 library, we have defined a combination of models, already included in the TALYS code, which better describes the production cross sections, for a variety of projectiles, incident energies and target masses.

The description of the optical, pre-equilibrium and level density models have been found to have a great influence on the calculated production cross section values. When proton, deuteron and alpha particle are used as projectile, better results are in general obtained using the optical model described, respectively by [12], [13] and [14]. And for these three projectiles, when a preequilibrium model based on the exciton model including numerical transition rates with optical model for collision probabilities [15] and a model for the microscopic level density from Hilaire's combinatorial tables [16] is used. The results of this combination of models are plotted in Figs. 1 to 4 as TALYS 1.6 Adj. Parametrizations of the models have not been changed. They are used as they are implemented in TALYS. Level density or optical model parameters haven't been tuned.

\section{Results and discussion}

TALYS version 1.6 results have been obtained using the default models (labelled Default) and using the combination of models (labelled Adj.) described in the section 2.3. In Figs. 1 to 4 , the full circles correspond to our experimental values, the other geometrical symbols to literature data, the full lines to TALYS Default and the dash lines to TALYS Adj. calculations. Four reactions, extracted from previous published articles, are presented. They cover proton, deuteron, alpha particle beams and a wide range of masses.

\subsection{The Th-226 production from the Th-232(p,3n)Pa-230 reaction}

Th-226 is a promising radionuclide for $\alpha$ RIT in leukaemia treatment. Th-226, with a half-life of 31 minutes, can be produced from the $\alpha$ decay of U-230, which has a half-life of 21 days, thanks to a generator system $\mathrm{U}-230 / \mathrm{Th}-226$. We studied the U-230 production using the Th-232(p,3n)Pa-230 $\left(\beta^{-}\right) \mathrm{U}-230$ reaction.

Our set of data for the Th-232(p,3n)Pa-230 reaction is plotted in Fig. 1. There is a good agreement with our experimental data and those available in the literature.

The TALYS code version 1.6 with default models and the TENDL-2015 library values, plotted with a dashedpointed line, are not able to reproduce the production cross section amplitude even if the shape is in good agreement 


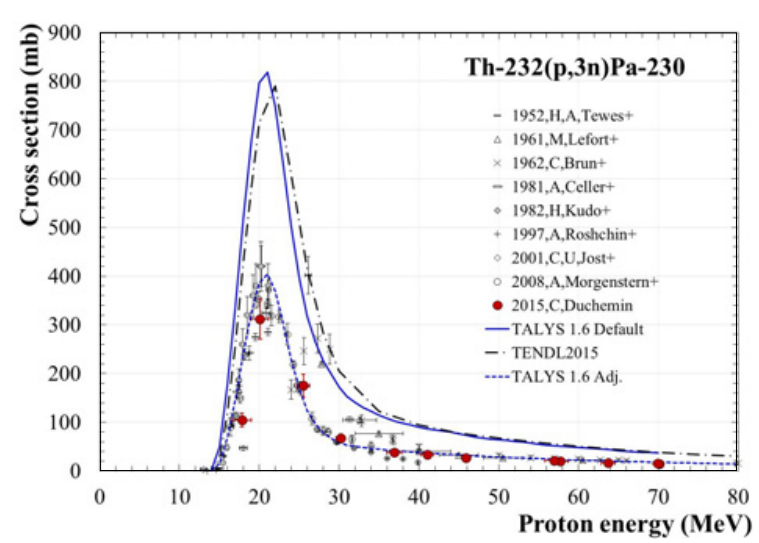

Figure 1. Th-232(p,3n)Pa-230 cross section.

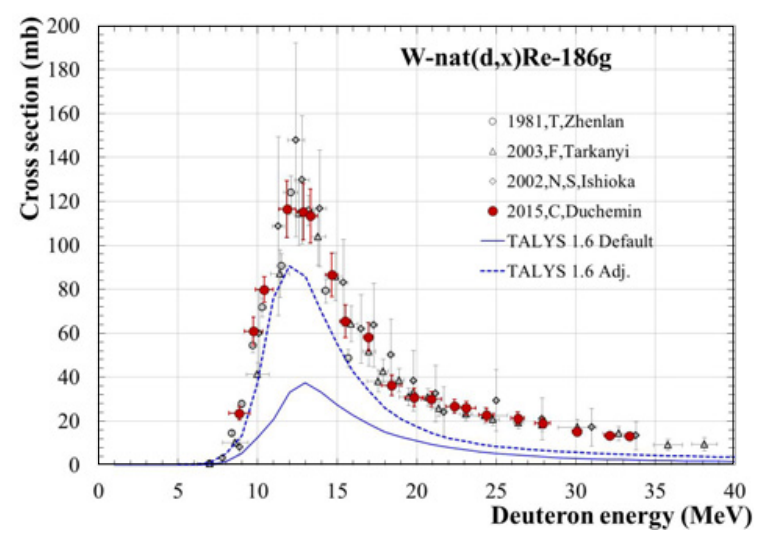

Figure 2. W-nat(d,x)Re-186g cross section.

with our experimental data. The TALYS 1.6 Adj. results are in perfect agreement with these experimental data.

\subsection{The Re-186g production from natural tungsten}

Re-186g, with a half-life of 3.7 days, is a $\beta^{-}$emitter used in clinical trials for the palliation of painful bone metastases resulting from prostate and breast cancer. It emits a $137 \mathrm{keV}$ gamma ray, suited for SPECT imaging.

The Fig. 2 shows that our experimental production cross section values are in agreement with the literature. The TALYS 1.6 Default calculation is far from the cross section amplitude. The TALYS $1.6 \mathrm{Adj}$. values are in better agreement but still underestimate the maximum of the cross section by $20 \%$.

\subsection{The $\mathrm{Sn}-117 \mathrm{~m}$ production from natural cadmium}

$\mathrm{Sn}-117 \mathrm{~m}$ is a conversion and Auger electron emitter, with a half-life of 13.6 days, useful for the palliation of painful bone metastases. Moreover, imaging can be achieved with its $158 \mathrm{keV}$ gamma line.

Our experimental production cross section values are in agreement with the values of Hermanne et al., published in 2010 (see Fig. 3).

However, the Qaim and Döhler values show a shift in energy, roughly $10 \mathrm{MeV}$ higher, for the location of the cross section's maximum. The points have been degraded rather far from the initial beam energy, which could explain the shift by energy straggling through the foils.

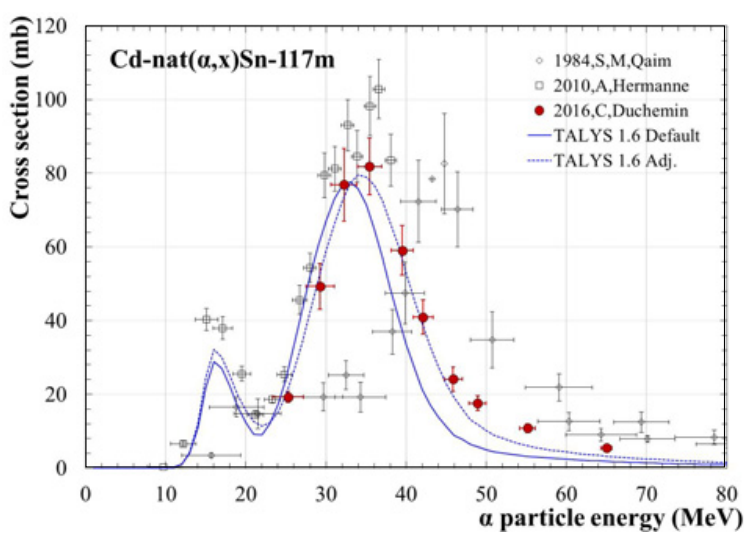

Figure 3. Cd-nat $(\alpha, \mathrm{x}) \mathrm{Sn}-117 \mathrm{~m}$ cross section.

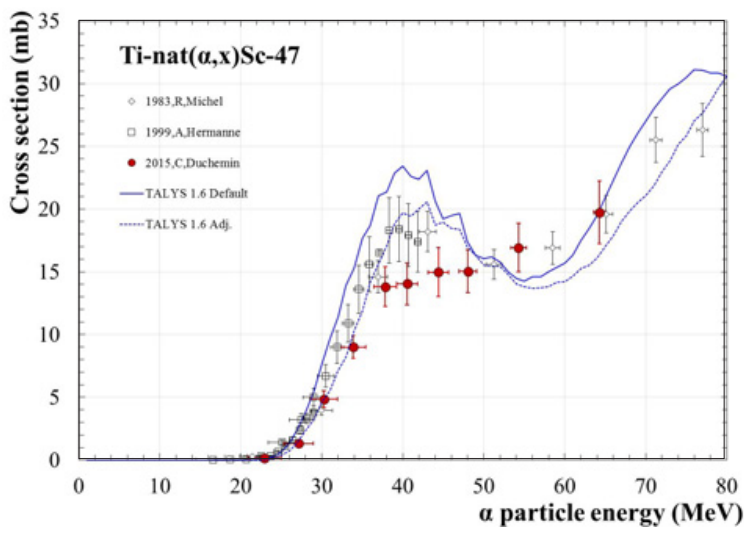

Figure 4. Ti-nat $(\alpha, \mathrm{x}) \mathrm{Sc}-47$ cross section.

The TALYS 1.6 Default models give the good shape but results are slightly underestimated and shifted in energy ( $3 \mathrm{MeV}$ lower). TALYS 1.6 Adj. models are in good agreement with the general behaviour of the experimental data. This combination allows reproducing the shape, the position of the maximum and the amplitude below $45 \mathrm{MeV}$.

\subsection{The Sc-47 production from natural titanium}

Sc-47, with a half-life of 3.35 days, is a $\beta^{-}$emitter of interest for a theranostic approach with Sc-44.

Our experimental production cross section values are plotted in Fig. 4. They are in agreement with the literature data sets even if the amplitude of the first maximum around $40 \mathrm{MeV}$ is lower.

The TALYS 1.6 Default calculation reproduces the right shape but with an overestimation of the amplitude below $50 \mathrm{MeV}$. TALYS 1.6 Adj. calculation is in good agreement with the general behaviour of the experimental data of A. Hermanne et al. reproducing well the shape and the amplitude.

\section{Conclusions}

A large set of production cross section of medical radionuclides have been collected using the stacked-foil technique with proton, deuteron and alpha beams delivered by the ARRONAX cyclotron and for various materials on a wide range of masses. Some comparisons have been systematically performed with the TALYS code, version 1.6. This code has been chosen because it includes a large number of theoretical models, the possibility to combine 
these models to better describe the experimental data and because the authors' team is very reactive and helpful. Three main mechanisms have been investigated, which have a great impact on the studied observables in our work. They are the optical potential, the level density description and the pre-equilibrium model. A set of models have been found allowing a good description of all our collected data, which is different from the suggested default combination of models. They can be used to get high quality data when no data are available in databases. Further investigations on other mechanisms that can affect our observable are ongoing.

The ARRONAX cyclotron is a project promoted by the Regional Council of Pays de la Loire financed by local authorities, the French government and the European Union. This work has been, in part, supported by a grant from the French National Agency for Research called "Investissementsd/Avenir", Equipex ArronaxPlus No. ANR-11-EQPX-0004 and Labex No. ANR- 11-LABX0018-01.

\section{References}

[1] F. Haddad, L. Ferrer, A. Guertin, T. Carlier, N. Michel, J. Barbet, J-F. Chatal, Eur. J. Nucl. Med. Mol. Imaging 35, 1377-1387 (2008)

[2] C. Duchemin, A. Guertin, F. Haddad, N. Michel, V. Métivier, Phys. Med. Biol. 60, 931-946 (2015)

[3] C. Duchemin, A. Guertin, F. Haddad, N. Michel, V. Métivier, Phys. Med. Biol. 60, 6847-6864 (2015)

[4] C. Duchemin, A. Guertin, F. Haddad, N. Michel, V. Métivier, Appl. Radiat. Isot. 97, 52-58 (2015)
[5] C. Duchemin, A. Guertin, F. Haddad, N. Michel, V. Métivier, Appl. Radiat. Isot. 118, 281-289 (2016)

[6] C. Duchemin, A. Guertin, F. Haddad, N. Michel, V. Métivier, Appl. Radiat. Isot. 115, 113-124 (2016)

[7] G. Blessing, W. Brautigam, H.G. Boge, N. Gad, B. Scholten, S.M. Qaim, Appl. Radiat. Isot. 955, 46-49 (1995)

[8] F. Tárkányi, S. Takács, K. Gul, A. Hermanne, M.G. Mustafa, M. Nortier, P. Oblozinsky, S.M. Qaim, B. Scholten, Yu.N. Shubin, Z. Youxiang, IAEATECDOC 1211, 49-152 (2001). Database available on https://www-nds.iaea.org/medportal/, update may 2013

[9] J.F. Ziegler, M.D. Ziegler, J.P. Biersack, Nucl. Instrum. Methods Phys. Res. B 268, 1818-1823 (2010)

[10] A.J. Koning, D. Rochman, Nucl. Data Sheets 113, 2841 (2012)

[11] A.J. Koning, D. Rochman, J. Kopecky, J. Ch. Sublet, E. Bauge, S. Hilaire, P. Romain, B. Morillon, H. Duarte, S. van der Marck, S. Pomp, H. Sjostrand, R. Forrest, H. Henriksson, O. Cabellos, S. Goriely, J. Leppanen, H. Leeb, A. Plompen and R. Mills, https://tendl.web.psi.ch/tendl_2015/ tendl2015.html

[12] A.J. Koning, J.P. Delaroche, Nucl. Phys. A 713, 231 (2003)

[13] Y. Han, Y. Shi, Q. Shen, Phys. Review C 73 (2006)

[14] P. Demetriou, C. Grama, S. Goriely, Nucl. Phys. A 707(1-2), 253-276 (2002)

[15] E. Gadioli, P.E. Hodgson, Oxford Univ. Press (1992)

[16] S. Goriely, S. Hilaire, A.J. Koning, Phys. Review C 78 (2008) 